\title{
Low Rates of Preemptive Kidney Transplantation: A Root Cause Analysis to Identify Opportunities for Improvement
}

\author{
Mona Alsharania, c, Faisal Basonbul ${ }^{\mathrm{a}}$, Seychelle Yohanna $^{\mathrm{b}}$
}

\begin{abstract}
Background: Kidney transplantation success can help improve the quality of life substantially and alleviate much of the physical, social and psychological stress that comes with dialysis lifestyle. Preemptive kidney transplantation has been established to be superior with more success rates than other means of transplantation types. However, low rates of this method need more attention to address the reason and tackle the root causes affecting low preemptive kidney transplantation.
\end{abstract}

Methods: This study was undertaken at a tertiary care academic hospital. We conducted a retrospective chart review of 50 living donor kidney transplants (recipient and donor charts) between January 1, 2017 and September 30, 2018 and performed a root cause analysis on the module. A three-step algorithm was developed to divide the root cause of all preemptive kidney transplantation.

Results: Out of 50 patients included, only $11(22 \%)$ achieved a successful preemptive kidney transplantation. Furthermore, an estimated glomerular filtration rate (eGFR) of 15 - 20 was a significant factor in achieving preemptive kidney transplantation $(\mathrm{P}=0.042)$. Meanwhile, 22 recipients started dialysis before transplant and was a major cause of not achieving the preemptive transplantation despite them achieving the status. Moreover, the most common recipient root cause was incidental medical issues in 10 cases $(20 \%)$ while in donor factors, the most common root cause was the delay in evaluating multiple donors to achieve the preemptive status $(20 \%)$.

Conclusion: Even though preemptive kidney transplantation is superior when compared to other modalities, achieving such a milestone is still considered very challenging. An eGFR of $15-20$ was a significant factor in achieving preemptive kidney transplantation, while pre-transplant dialysis was a major cause of not achieving it. Further large-scale studies are needed to identify new factors as well as vali-

Manuscript submitted November 9, 2020, accepted December 11, 2020

Published online January 12, 2021

aNephrology Department, McMaster University, Hamilton, Ontario, Canada ${ }^{b}$ Renal Transplant Department, McMaster University, Hamilton, Ontario, Canada

${ }^{\mathrm{c} C o r r e s p o n d i n g ~ A u t h o r: ~ M o n a ~ A l s h a h r a n i, ~ N e p h r o l o g y ~ D e p a r t m e n t, ~ M c M a s-~}$ ter University, St. Joes Hospital, 50 Charlton Ave. E, L8s4L8, Hamilton, Ontario, Canada. Email: mona.alshahrani@medportal.ca

doi: https://doi.org/10.14740/jocmr4391 date our findings.

Keywords: Preemptive kidney transplantation; Root cause; Recipient factors; Donor factors

\section{Introduction}

Preemptive kidney transplantation is defined as receiving transplant before the deterioration of the kidney and before the initiation of the dialysis [1]. It is considered the best form of renal replacement therapy resulting in lower morbidity and mortality and improved quality of life [2]. Preemptive kidney transplantation has a variety of advantages over transplantation after a course of maintenance dialysis therapy. This includes fewer pre-transplant blood transfusions, increased rates of patients continuing employment, improved long-term graft survival, lower rates of delayed graft function and fewer episodes of acute rejection [3]. Despite these recognized benefits, the utilization of preemptive kidney transplantation remains low, with reported rate of $12 \%$ of all recipients receiving a kidney transplant in United States $[2,3]$.

Patient with kidney failure has to identify a living donor which is difficult for many patients since they will not be on the transplant list until they develop a chronicity of the disease that did not respond to either medical or surgical treatments [4]. Therefore, patients commonly refuse to approach their loved ones for fear of a negative health outcome [5]. Moreover, potential donors may be deterred from coming forward due to costs such as transportation, childcare and lost income from time off work [6]. Furthermore, the transplant candidates have to withstand a transplant program at an appropriate time and undergo a lengthy process to determine transplant eligibility [6]. There is no national benchmark for the length of time to complete a recipient or donor evaluation; however, most transplant programs agree that in both cases, the goal is between 6 and 12 months [7]. The Canadian Society of Transplantation recommends candidates be referred early when the glomerular filtration rate (GFR) is less than $20 \mathrm{~mL} / \mathrm{min} / 1.73 \mathrm{~m}^{2}$ with evidence of progressive and irreversible deterioration in renal function over the previous $6-12$ months, the maximum time needed for the candidate to be cleared [8]. However, if a patient has not been able to identify a potential living donor at that time, then referral for transplant early is not optimal resourcefully. Therefore, predicting when a patient's kidney 


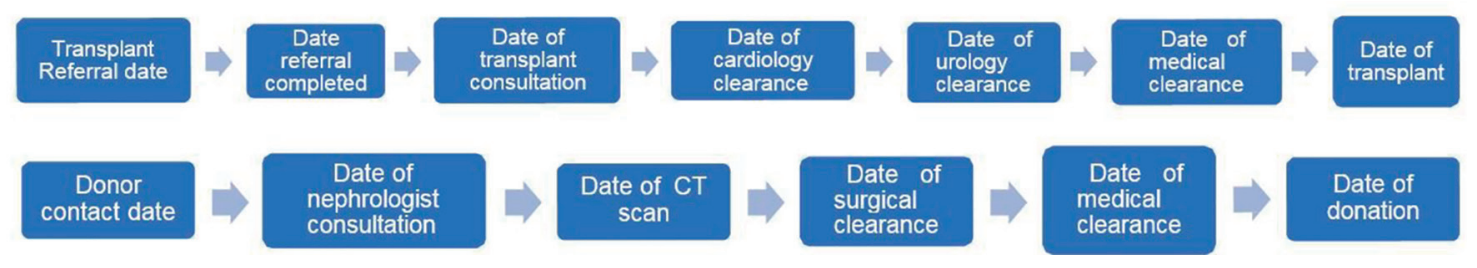

Figure 1. Recipient and donor evaluation process.

function will reach that critical level where transplantation would be optimal can be prone to error for obvious reasons [9].

The St. Joseph's Healthcare Hamilton (SJHH) kidney transplant program performs approximately 30 living donor kidney transplants each year and only $20 \%$ of these are preemptive transplants. $\mathrm{SJHH}$ is committed to improving the proportion of recipients who achieve preemptive kidney transplantation and would like to make programmatic changes to achieve this $[10,11]$. However, the root causes of the problem have not been thoroughly identified and appreciated in our local context. A root cause analysis is a systematic approach aimed at discovering the causes of a healthcare problem, with a focus on systems issues [11]. Utilizing this methodology helps to determine the presence of quality gaps in this complex process with many moving parts. Our goal was to identify modifiable program-related barriers for future quality improvement opportunities aimed at increasing the rates of preemptive kidney transplantation.

\section{Materials and Methods}

This study was undertaken at a tertiary care academic hospital serving a health region of approximately 721,000 people. The SJHH kidney transplant program receives referrals from five regional chronic kidney disease programs, including one located at SJHH, Hamilton, Ontario, Canada. We conducted a retrospective chart review of 50 living donor kidney transplants (recipient and donor charts) between January 1, 2017 and September 30, 2018 and performed a root cause analysis on the module. Ethical approval from the Hamilton Integrated Research Ethics Board (HiREB) was obtained. This study was conducted in compliance with all the applicable institutional ethical guidelines for the care, welfare and use of animals.

\section{Inclusion and exclusion criteria}

We included all donors who were ultimately reviewed as an eligible candidate in cases where some recipients having more than one candidate were found. In addition, all recipients having one sole candidate were included when found eligible. Regarding exclusion criteria, no transplant was excluded from the data.

\section{Data collection}

In-depth interviews with the transplant and donor coordinators were conducted to understand the recipient and donor evalu- ation processes and the required testing and consultations to receive clearance for surgery (Fig. 1). Two independent reviewers (MA and FB) extracted data from charts and reviewed each chart extensively. If more clarity was needed regarding a specific case, the transplant and donor coordinators were consulted. Charts of both recipients and donors were reviewed to examine whether delays in the evaluation occurred. Basic demographic information was collected (age, cause of end-stage renal disease (ESRD) and home renal program). We recorded the dates of each milestone in the evaluation. For a transplant recipient, we recorded the date of referral, transplant nephrologist appointment, dialysis start date for recipients who started dialysis prior to transplantation, cardiology clearance, urology clearance, final medical clearance, transplant date and estimated GFR (eGFR) at the time of referral for those who referred preemptively. For donors, we collected the date of donor contact to the donor program, donor nephrologist appointment, computed tomography (CT) scan, surgical clearance and final clearance. Part way through the study, it was felt that the time to complete initial blood work could be a cause of significant delay; therefore for a subset of 20 donors and recipients, the date the initial laboratory investigations were completed was collected. All of the evaluation times were set as continuous variables represented as median and range.

\section{Root cause and statistical analysis}

We divided the possible barriers to achieving a preemptive kidney transplant into the following broad categories: program, provider, recipient and donor factors. After conducting a literature search and interviews with healthcare providers, we identified potential causes under each category (Fig. 2). Using a fishbone diagram approach gave us confidence that we had considered all potential root causes for the next stage of the study. Afterwards, we conducted an in-depth analysis of the charts of both donors and recipients to identify the top two contributing factors. In a traditional root cause analysis, only one root cause is usually identified. However, the process to achieve a preemptive kidney transplant was quite complex where assigning a single root cause may not be appropriate in some cases. For example, regardless of the primary root cause, we examined eGFR at the time of referral since a lower eGFR usually represents less time for a donor and recipient to be successful at achieving a preemptive kidney transplant. We defined late referral as having an eGFR less than 15 at the time of referral. Our data were analyzed using SSPS, version 26 (IBM, Armonk, NY). P value of less than 0.05 was considered to be statistically significant. Categorical variables were repre- 


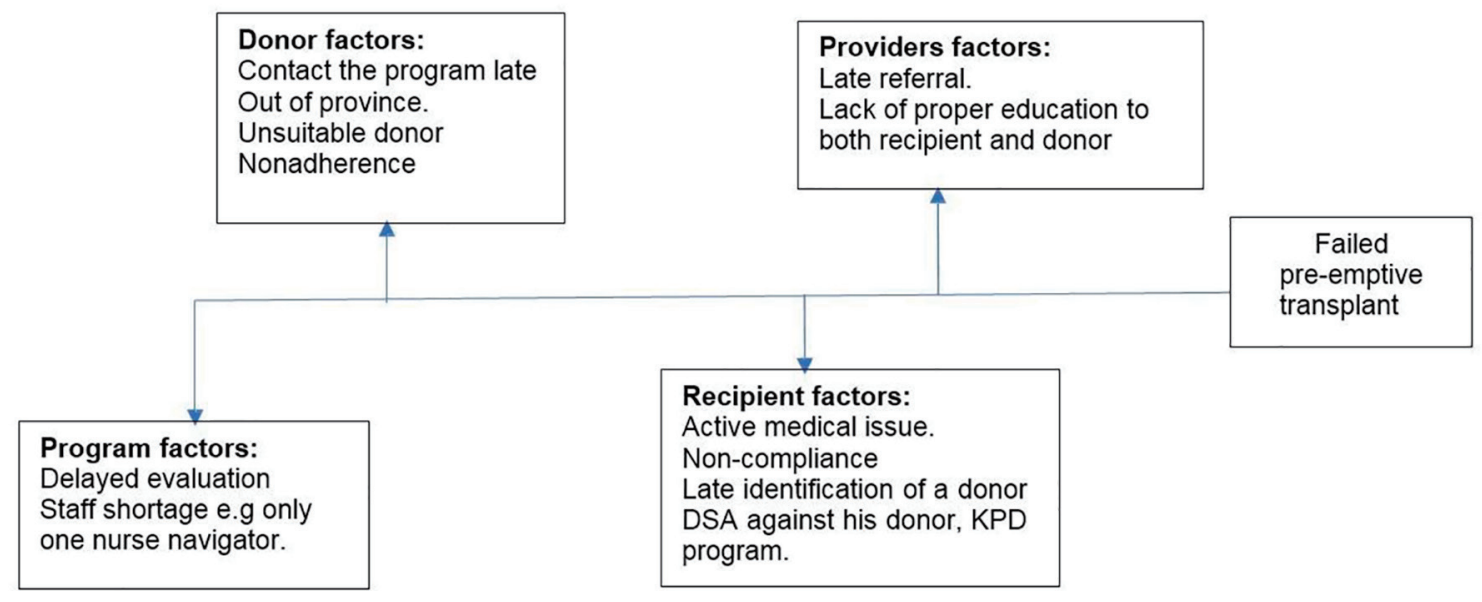

Figure 2. Fishbone diagram illustrating potential barriers to preemptive kidney transplant. DSA: donor-specific antibody; KPD: kidney paired donation.

sented as frequencies, and percentages with Chi-square test (or Fisher's exact test, as appropriate) were used for testing the advertisement platform differences. For continuous variables, the representation was as means and standard deviations or medians and ranges, using skewness and Kurtosis tests to evaluate the normal distribution of the variables. Based on normality status, independent samples $t$-test or Mann-Whitney $\mathrm{U}$ test was used to compare patients on basis of achieving preemptive transplant. We developed an algorithm to assign root causes for each case of a failed preemptive kidney transplant. The algorithm is as follows: STEP 1: "A delayed identification of a living donor" as a root cause was determined if the recipient was already on dialysis, at the time of referral. However, in order to determine whether the nephrologist, the patient or both of them was the cause of the problem, we had to interview them both and review the chronic kidney disease (CKD) clinic documentation. STEP 2: 1) In order to consider that a recipient is considered a preemptive kidney transplant case, both recipients and donors needed to complete their evaluation process at least 8 weeks before dialysis would be a requirement to provide time for surgical clearance and planning. 2) If the previously mentioned condition was met but a preemptive kidney transplant was not achieved, our next step was to examine whether the recipient and donor pair was ABO or HLA incompatible necessitating entry into the national kidney paired donation program (KPD) delaying time to transplant as well as the eGFR levels at the time of referral leading to two root cause scenarios: First, if the pair had to enter KPD and the eGFR was less than 15 , we attributed root cause as "late referral and required KPD". Second, if the eGFR was greater than or equal to 15 and the pair were medically cleared but required KPD, we attributed root cause as "required KPD", even if the eGFR of $>15$ was achieved but there was a multiple unmatched rounds of KPD leading to a failed preemptive transplantation. STEP 3: If one of the primary preconditions was not met (i.e. either the recipient or the donor had not completed their evaluation at least 8 weeks prior to the requirement of dialysis), the root cause scenarios were determined as following: 1) "Late referral" as a root cause is when a recipient was not cleared for surgery at least 8 weeks prior to requiring dialysis (with a donor who was approved for surgery) and their eGFR was below 15 at the time of referral, and they required initiation of dialysis within 6 months of being referred. 2) "Acute medical/social" root cause, if an acute medical or social issue arose during the evaluation process (e.g. stroke, new malignancy) that lengthened the time to complete the evaluation of either the donor or the recipient. 3) "Delay in recipient evaluation" root cause is when the time to complete certain milestones of the evaluation was long despite not having any obvious clinical cause. 4) "Required multiple donors to be evaluated" as a root cause, if the initial donor was deemed ineligible and multiple donors needed to be evaluated before one was approved for donation. 5) "Delay in donor evaluation" as a root cause, if we established the time to complete certain milestones of the evaluation for the donors and the time to complete a milestone was long despite there being no obvious clinical cause. 6) "Late donor contact" as a root cause, if the first donor did not contact the program early enough in the process to allow enough time to complete the evaluation prior to their recipient requiring dialysis.

\section{Results}

In regard to the descriptive data, we divided our cohort of recipients for the purpose of analysis into preemptive and nonpreemptive recipients (Table 1). Both groups were predominantly male with a higher proportion in the non-preemptive group (55\% vs. 69\%, respectively). The mean age was higher in the non-preemptive group ( $50 \pm 16$ years), while the mean age in the preemptive group was $46 \pm 15$ years. The predominant cause of ESRD in our cohort was glomerulonephritis in both preemptive group (45\%) and failed preemptive group (38\%). On the other hand, the mean age in the preemptive group was 45 years versus 47 years in the non-preemptive group, while the majority of donors were male in both groups $(60 \%$ versus $63 \%$ ). Meanwhile, in the majority of cases, the actual donor was the first donor to be evaluated $(64 \%)$ in both groups. There was a higher proportion of spousal donors in the preemptive 
Table 1. Demographics and Baseline Characteristics

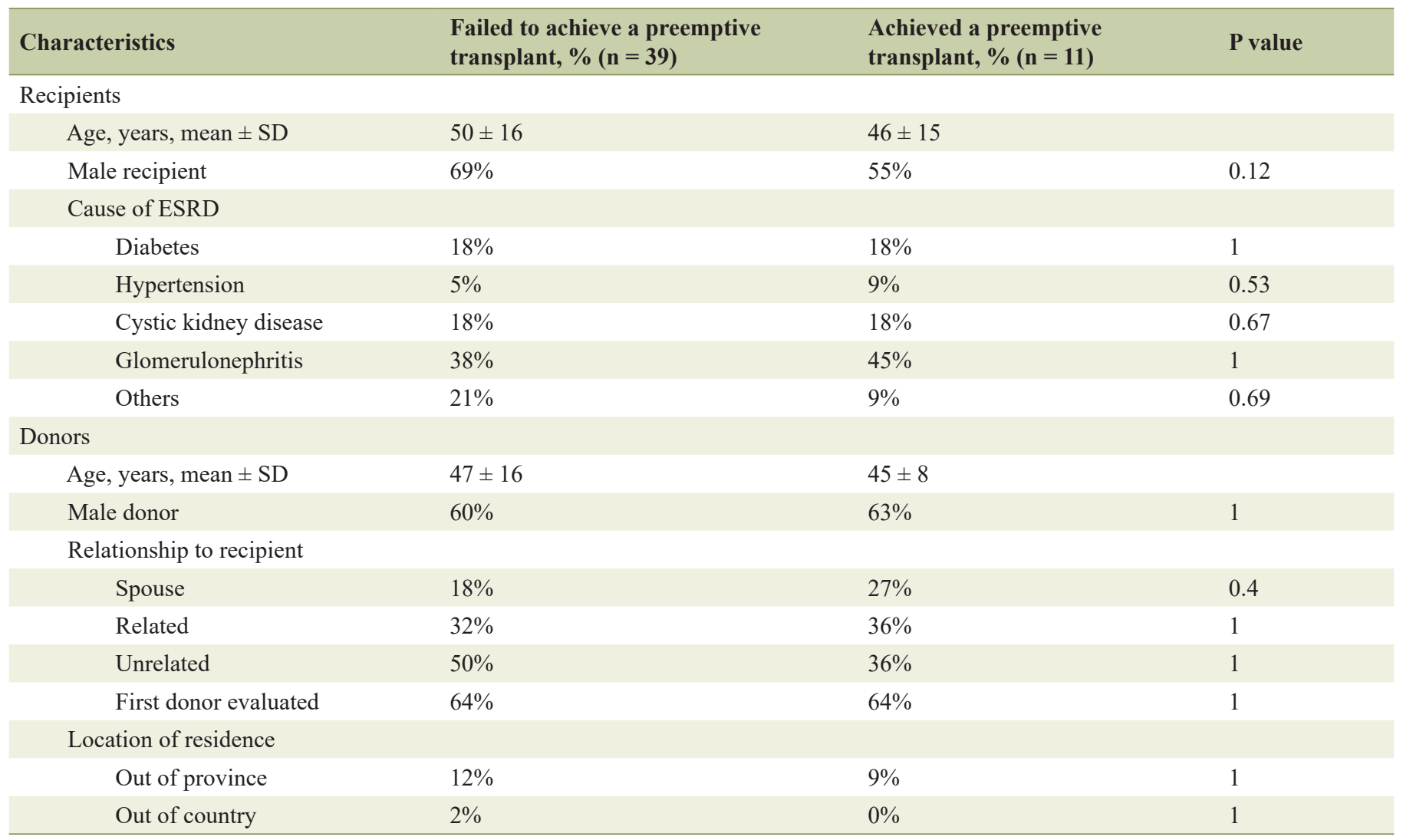

ESRD: end-stage renal disease.

group compared to non-preemptive group ( $27 \%$ versus $18 \%$ ). In contrast, unrelated non-spousal donors were more common in the non-preemptive group (50\% versus $36 \%$ ) (Table 1 ).

Table 2 summarizes the eGFR of the preemptive group of recipients at the time of referral broken down by categories of eGFR as following: less than 15, 15 - 20 and more than 20 , similar to the KDIGO classification. Furthermore, 55\% of those who achieved a preemptive kidney transplant had an eGFR between 15 and 20 at the time of the referral $(P=0.049)$. In contrast, $77 \%$ of those who failed to achieve the preemptive transplant had eGFR of less than 15 at time of referral $(\mathrm{P}=0.052)$. This was consistent with the results of logistic regression model where eGFR of 15 - 20 had higher odds of achieving a preemptive kidney transplant, compared to those with eGFR less than 15 (odds ratio (OR): 9.71; 95\% confidence interval $(\mathrm{CI}): 1.08-87.31 ; \mathrm{P}=0.042)$. In the same con- text, eGFR values greater than 20 were associated with lower odds of achieving a preemptive kidney transplant, compared to those with eGFR less than 15 (OR: 0.14 ; 95\% CI: 0.02 $0.84 ; \mathrm{P}=0.032$ ). However, on adjusting the results for age, sex and cause of the ESRD, the significance persisted only for eGFR values greater than 20 (OR: 0.03 ; 95\% CI: 0.00 - 0.34; $\mathrm{P}=0.005)$, while it faded away for the eGFR of $15-20$ group (OR: 10.69; 95\% CI: $0.92-124.91 ; \mathrm{P}=0.059$ ). This change in significance may be driven by the small sample size limitation (Table 3).

Regarding milestones, we carefully reviewed each chart for the time to complete each major evaluation milestone. In the recipient group, we found that the median time from referral to clearance was 408 days. The median time from referral to completion of pre-referral testing was 34 days. The median time from pre-referral testing to transplant nephrology consul-

Table 2. eGFR at Time of Referral for Recipients Referred Preemptively Only

\begin{tabular}{|c|c|c|c|}
\hline eGFR $\left(\mathrm{mL} / \mathrm{min} / 1.73 \mathrm{~m}^{2}\right)$ & $\begin{array}{l}\text { Failed to achieve a preemptive } \\
\text { transplant, } \%(n=39)\end{array}$ & $\begin{array}{l}\text { Achieved a preemptive kidney } \\
\text { transplant, } \%(n=11)\end{array}$ & $P$ value \\
\hline Less than 15 & $77.3 \%$ & $36 \%$ & 0.052 \\
\hline Greater than 20 & $4.5 \%$ & $9 \%$ & 1 \\
\hline
\end{tabular}

eGFR: estimated glomerular filtration rate. 
Table 3. Logistic Regression Model of eGFR Values and Odds of Achieving Preemptive Transplant*

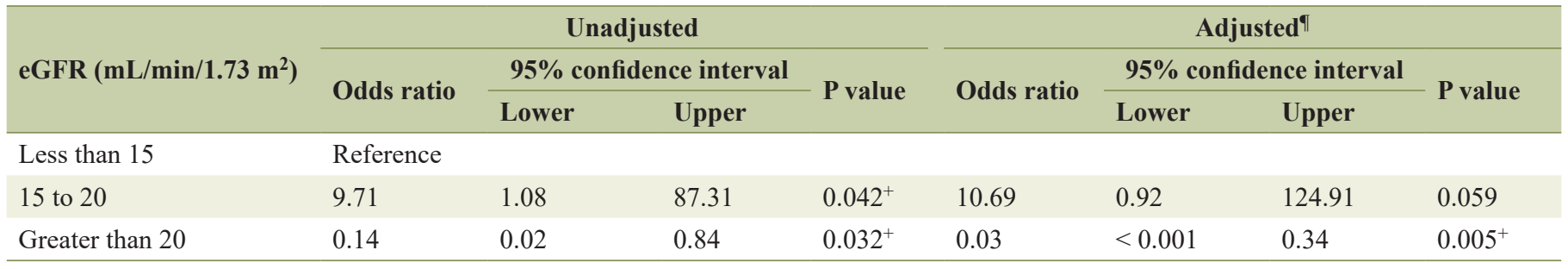

*Represent the odds of "preemptive kidney = achieved" vs. "preemptive kidney = failed". " Adjusted for age, sex and cause of ESRD. +Statistically significant. eGFR: estimated glomerular filtration rate.

tation was 38 days. The median time from transplant nephrology consultation to final clearance for surgery was 324 days. Lastly, the median time from final clearance to transplantation was 90 days (Table 4).

In the donor group, the median time from donor evaluation questionnaire completion to final clearance was 241 days. The median time between questionnaire completion and the completion of pre-consultation testing was 44 days. The median time from pre-consultation testing completion to the donor nephrologist appointment was 49 days. Donor clearance also took a median of 120 days from the initial donor consultation appointment. Interestingly, nine cases of donor clearance occurred after recipient clearance. Root cause breakdown was performed on all 50 transplant cases. Only 11 recipients $(22 \%)$ achieved a preemptive kidney transplant while in the remaining 39 recipients, 17 (34\%) were already on dialysis at the time of referral, and therefore the root cause was attributed to late referral or late identification of a living donor. Twenty-two (44\%) others were referred preemptively but failed to achieve preemptive transplant (Fig. 3). In four cases (8\%), entry into KPD was labeled as a root cause as both recipient and donor pair were cleared for transplant prior to the recipient initiating dialysis. For recipient factors, the most common root cause encountered was incident medical issues $(20 \%)$ which occurred in 10 recipients (four recipients had new malignancies identified during screening, two required nephrectomies, two had unprovoked pulmonary embolism, one cerebrovascular accident and one acute infection). Social issues were implicated in two cases (4\%) due to multiple missed appointments. Finally, for donor factors, the need to evaluate multiple donors prior to one being approved for surgery was implicated as either primary or secondary root cause in 10 cases $(20 \%)$. There were delays in donor evaluation in four donors $(8 \%)$. These were divided equally between donor non-compliance with pre-consultation or pre-clearance testing and the requirement of further medical subspecialty assessments prior to clearance for transplant.

\section{Discussion}

Our study represents one of the few applications of root cause breakdown in an attempt to identify reasons for failed preemptive transplantation in patients with CKD. It is also the first

Table 4. Time to Complete Evaluation Milestones for Recipients and Donors

\begin{tabular}{|c|c|c|}
\hline & Median (days) & Range \\
\hline \multicolumn{3}{|l|}{ Recipient milestones $(\mathrm{n}=50)$} \\
\hline Referral to initial testing & 34 & $4-200$ \\
\hline Consultation to recipient clearance & 324 & $63-1,945 \dagger$ \\
\hline Recipient clearance to transplantation & 90 & $22-1,176 \dagger$ \\
\hline \multicolumn{3}{|l|}{ Donor milestones $(\mathrm{n}=50)$} \\
\hline Questionnaire returned to initial testing & 44 & $5-403$ \\
\hline Initial testing completion to donor nephrologist consultation & 49 & $5-136$ \\
\hline Donor nephrologist consultation to donor clearance & 120 & $7-255$ \\
\hline Donor questionnaire returned to donor clearance & 241 & $61-506$ \\
\hline
\end{tabular}

†Three polycystic kidney disease recipients had longer wait times between initial assessment and clearance due to delayed nephrectomies just prior to reaching end-stage renal disease and transplant. ${ }^{*}$ The time interval between recipient referral and their donor completing the donor questionnaire. ${ }^{\wedge}$ The negative result in this range is due to five donors who contacted the program prior to their respective recipient's referral. Also, the range is affected by whether multiple donors contacted the program at varying stages before a suitable donor was found. 


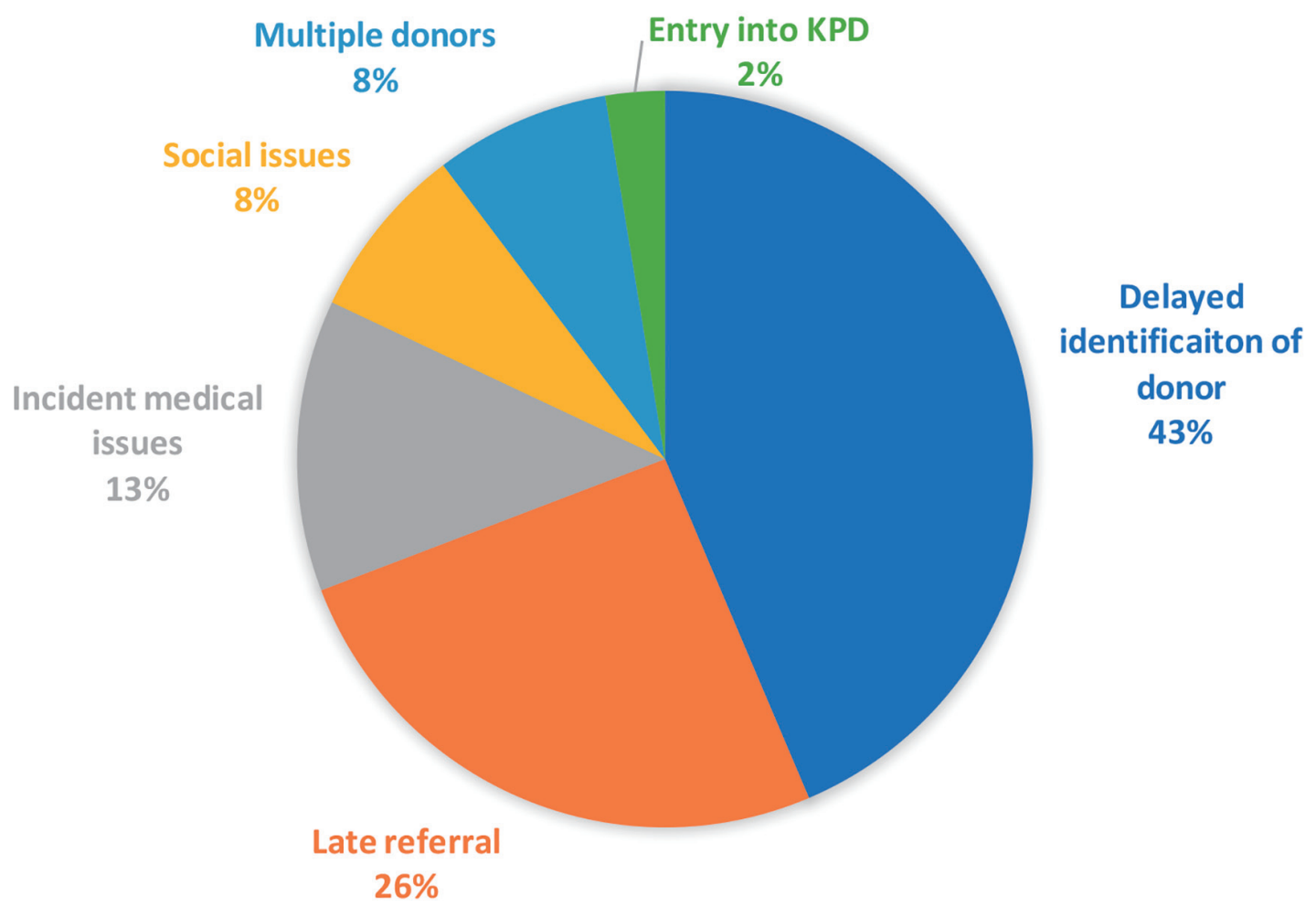

Figure 3. Distribution of root causes amongst non-preemptive transplant recipients $(n=39)$. KPD: kidney paired donation.

study that investigated this critical topic in the context of the Canadian healthcare system where personal financial constraints have a smaller impact due to the availability of universal healthcare. Furthermore, it was found that late referral was the most frequently identified cause for failing to achieve a preemptive transplant. This is especially important since the timing of referral for transplant is a potentially modifiable practice. In other words, our study suggests that an earlier referral, as well as earlier identification of potential donors, could lead to more preemptive transplants. This is supported by the fact that the mean eGFR at referral in those who did achieve preemptive transplant was much higher, as it allowed for more time to achieve our goal. Moreover, it is also revealed that there were extensive delays in both the recipient and donor evaluation process. Other factors, such as the discrepancy between timing of recipient referral and donor contact, delays in completion of initial workup and the lack of clear prioritization for recipients in other subspecialty consults or imaging studies were significant factors.

Despite the well-known positive health outcomes from preemptive kidney transplantation, several publications have shown that this does not appear to be particularly emphasized during modality education $[12,13]$. Coorey et al found that $60 \%$ of surveyed patients viewed transplantation as a last resort and half the respondents believed that transplantation would only be a consideration after dialysis while half the respondents attributed the success to donation to education [14]. Despite preemptive being underutilized whether due to lack of identification or other factors, it has been shown to be superior in many factors such as allograft survival rate and avoiding long wait time for donor match [15]. Gill et al reported an increase rate of allograft survival rate as well as a decrease in death rate in those who had preemptive transplant compared to those who did not by $31 \%$ [16]. Lack of awareness of the assessment process and the required steps to be approved for transplant could lead to a lack of motivation to complete testing. It can also be noted that once patients experience dialysis, the realization of its associated burden on both health and quality of life would fuel the desire for transplantation assessment, although we did not focus on this area specifically in this study. These delays are also compounded by having no clear expedited protocol for this unique subset of our recipients in regards to imaging studies and the difficulties with identification of which stage the patient is currently at in the transplant process by the various members of the healthcare team outside of the transplant nephrology group.

As explained earlier, we believe the uniqueness of the chosen study population as well as more in-depth root cause analysis and milestone identification lend significant strength to our study. Our choice of relatively more recent transplantations is also more reflective on the current state of the assessment process and easier application of potential quality improvement projects in the future.

Our study did have several important limitations. The switch to a new electronic medical record system (EMR) at our center and the significant changes to the referral acceptance process, both of which were introduced in late 2017, resulted in a limitation in the number of available recipients to study. 
These included a new requirement for completion of pre-referral testing prior to receiving an appointment for consultation as well as prioritization of recipients with identified donors who had already contacted the program. Also, patient information and assessments remain split between a paper chart and the EMR. And, as many of these assessments were initiated years earlier, it was often difficult to ascertain when certain milestones occurred, e.g. exact date of initiation of referral, date of completion of initial workup, date of clearance. We attempted to circumvent this by using the most reasonable approximation of such dates, e.g. dates the referral package was received by the transplant coordinator, dates of blood work receipt, dates of the completion of the last necessary investigations. We believe these were fair proxies that would still lend to productive analysis. In addition, there are no established cut-offs in the literature for what level of eGFR constitutes a late referral or a delay in donor contact or completion of evaluation and we used what we considered reasonable values given the median duration between referral and initiation of dialysis. We have also used eGFR in assessing referral timing while the 2-year risk of progression to ESRD based on the kidney failure risk equation is the traditional tool used for timing of renal replacement therapy in the CKD clinic. Improvements in the donor assessment process with the recent introduction of the "one-day donor assessment clinic" in our program should significantly reduce assessment times especially when multiple donors need to be assessed. For the patients who were referred already on a dialysis, which accounted for $34 \%$ of our cohort, we did not pursue further analysis into the causes as that was not deemed the primary goal of this study. As previously mentioned, referrals for recipients already on dialysis may represent a late referral or late identification of a donor. We were not able to ascertain this from our study. Finally, we did not conduct a statistical analysis comparing recipients in the preemptive and non-preemptive group; therefore we could not confidently comment on the differences in the two groups. This will be the next step in our project.

\section{Conclusion}

We believe our study did identify important areas for program improvement. We identified the potential for future quality improvement projects focusing on the transplant referral process and opportunities to streamline the recipient and donor evaluation processes (e.g. expedited imaging studies, easier access to subspecialty consultation to expedite recipient clearance). An eGFR of 15 - 20 was a significant factor in achieving preemptive kidney transplantation, while pre-transplant dialysis was a major cause of not achieving it. Future studies into the timing of modality education and transplant education in the CKD clinic are needed as well.

\section{Acknowledgments}

We appreciate the support of McMaster University and all those who participated in the study for their time and effort.

\section{Financial Disclosure}

There was no funding.

\section{Conflict of Interest}

The authors declare that they have no conflict of interest.

\section{Informed Consent}

All subjects provided written informed consent.

\section{Author Contributions}

MA, FB and SY contributed equally into designing, analyzing, writing and proofreading the manuscript. In addition, MA and FB were responsible for data collecting, reference collection, reference checking and final language editing of the manuscript.

\section{Data Availability}

The authors declare that data supporting the findings of this study are available within the article.

\section{References}

1. Sayin B, Colak T, Tutal E, Sezer S. Comparison of preemptive kidney transplant recipients with nonpreemptive kidney recipients in single center: 5 years of followup. Int J Nephrol Renovasc Dis. 2013;6:95-99.

2. Helmick RA, Jay CL, Price BA, Dean PG, Stegall MD. Identifying barriers to preemptive kidney transplantation in a living donor transplant cohort. Transplant Direct. 2018;4(4):e356.

3. Canadian Organ Replacement Register. 2019. Available from: https://www.cihi.ca/en/canadian-organ-replacementregister-corr.

4. Kiberd BA, Tennankore KK, West K. Eligibility for the kidney transplant wait list: a model for conceptualizing patient risk. Transplant Res. 2014;3(1):2.

5. Davis LA, Grogan TM, Cox J, Weng FL. Inter- and intrapersonal barriers to living donor kidney transplant among black recipients and donors. J Racial Ethn Health Disparities. 2017;4(4):671-679.

6. Nakamura T, Ushigome H, Nakao T, Harada S, Koshino $\mathrm{K}$, Suzuki T, Ito T, et al. Advantages and disadvantages of pre-emptive kidney transplantation: results from a single transplantation center. Transplant Proc. 2015;47(3):626629.

7. Rudow DL, Swartz K, Phillips C, Hollenberger J, Smith T, Steel JL. The psychosocial and independent living donor advocate evaluation and post-surgery care of living 
donors. J Clin Psychol Med Settings. 2015;22(2-3):136149.

8. Habbous S, McArthur E, Sarma S, Begen MA, Lam NN, Manns B, Lentine KL, et al. Potential implications of a more timely living kidney donor evaluation. Am J Transplant. 2018;18(11):2719-2729.

9. Grams ME, Massie AB, Coresh J, Segev DL. Trends in the timing of pre-emptive kidney transplantation. J Am Soc Nephrol. 2011;22(9):1615-1620.

10. LaPointe Rudow D, Hays R, Baliga P, Cohen DJ, Cooper M, Danovitch GM, Dew MA, et al. Consensus conference on best practices in live kidney donation: recommendations to optimize education, access, and care. Am J Transplant. 2015;15(4):914-922.

11. Charles R, Hood B, Derosier JM, Gosbee JW, Li Y, Caird MS, Biermann JS, et al. How to perform a root cause analysis for workup and future prevention of medical errors: a review. Patient Saf Surg. 2016;10:20.

12. R LR, Isherwood L, Ben-Tovim D. Why do older peo- ple with multi-morbidity experience unplanned hospital admissions from the community: a root cause analysis. BMC Health Serv Res. 2015;15:525.

13. Abecassis M, Bartlett ST, Collins AJ, Davis CL, Delmonico FL, Friedewald JJ, Hays R, et al. Kidney transplantation as primary therapy for end-stage renal disease: a National Kidney Foundation/Kidney Disease Outcomes Quality Initiative (NKF/KDOQITM) conference. Clin J Am Soc Nephrol. 2008;3(2):471-480.

14. Czyzewski L, Wyzgal J. The adequacy of transplantation education in the ESRD population in Poland. Ann Transplant. 2012;17(2):62-73.

15. Coorey GM, Paykin C, Singleton-Driscoll LC, Gaston RS. Barriers to preemptive kidney transplantation. Am J Nurs. 2009;109(11):28-37; quiz 38.

16. Gill JS, Tonelli M, Johnson N, Pereira BJ. Why do preemptive kidney transplant recipients have an allograft survival advantage? Transplantation. 2004;78(6):873879. 